\title{
Diabetic Ketoacidosis Following Administration of Cervical Epidural Steroid Injection in a Non-Diabetic
}

\author{
Andrew C. Berry ${ }^{\mathrm{a}, \mathrm{h}}$, Matthew E. Tick ${ }^{\mathrm{a}}$, Brijesh B. Patel ${ }^{\mathrm{b}}$, Cheryl W. O'Malley ${ }^{\mathrm{c}}$, Rahman Nakshabendid, \\ Jason Bellardini ${ }^{\mathrm{d}}$, Ariel Caplan ${ }^{\mathrm{e}}$, Nick A. Berry ${ }^{\mathrm{f}}$, Warren L. Reuther III ${ }^{\mathrm{g}}$
}

\begin{abstract}
Our case describes a 56-year-old African-American female who underwent fluoroscopy-guided epidural steroid injection for recurrent neck and arm pain. The administration of steroid injections has been shown to diminish peripheral glucose metabolism and to stimulate gluconeogenesis, thus elevating blood glucose concentration and worsening insulin resistance. Though uncommon, diabetic ketoacidosis (DKA) can arise following both epidural steroid treatments in diagnosed diabetics or in cases of coinciding infection. At the time of the epidural injection, the patient showed no signs of acute infection, concomitant drug use or any documented diabetes, all potential causes of DKA. Our case is unique because it describes a documented non-diabetic patient who went into DKA following cervical epidural steroid injection.
\end{abstract}

Keywords: Diabetic ketoacidosis; Diabetes; Epidural steroid injection; Degenerative disc disease

\section{Introduction}

Degenerative disc disease of the cervical spine is both a

Manuscript accepted for publication July 4, 2014

${ }^{a}$ Kansas City University of Medicine and Biosciences, Kansas City, MO, USA

${ }^{b}$ Department of Internal Medicine, University of South Florida, Tampa, FL, USA

${ }^{\mathrm{c}}$ Department of Internal Medicine, Banner Good Samaritan Medical Center, University of Arizona College of Medicine-Phoenix, Phoenix, AZ, USA

${ }^{\mathrm{d}}$ Department of Internal Medicine, University of Florida-Jacksonville, Jacksonville, FL, USA

${ }^{\mathrm{e}}$ Department of Internal Medicine, Palm Beach Centre for Graduate

Medical Education, West Palm Beach, FL, USA

${ }^{\mathrm{f}}$ The Ohio State University, Columbus, OH, USA

${ }^{\mathrm{g}}$ Department of Radiology, West Palm Hospital, West Palm Beach, FL, USA

${ }^{\mathrm{h}}$ Corresponding Author: Andrew C. Berry, Kansas City University of Medicine and Biosciences, 1750 Independence Ave, Kansas City, MO 64106, USA. Email: ABerry5555@gmail.com

doi: http://dx.doi.org/10.14740/jmc1846w debilitating and rising problem among the elderly [1]. If patients fail conservative medical and physical therapy, epidural steroid injections are considered prior to surgical evaluation. Fluoroscopy-guided cervical epidural injections are safe and efficacious [2], but do come with a myriad of possible complications such as headaches, epidural hematoma, infection, injection site pain, paraplegia, vasovagal reactions, elevated glucose levels and insulin resistance [3, 4]. The administration of steroid injections has been shown to dampen peripheral glucose metabolism and to stimulate gluconeogenesis, thus elevating blood glucose concentration and worsening insulin resistance [5]. Thus, diagnosed diabetics run the risk of glucose imbalance following epidural steroid injection. Though uncommon, patients may even present with hyperglycemia, ketonemia, confusion and anion gap metabolic acidosis, all signs of diabetic ketoacidosis (DKA). Our case is unique because it describes a confirmed non-diabetic patient who went into DKA following cervical epidural steroid injection for chronic neck pain.

\section{Case Report}

A 56-year-old African-American female presented to her primary care physician complaining of neck pain and right arm pain of insidious onset that radiated down her arm. The patient's past medical history was notable for hypertension, hyperlipidemia and cerebrovascular accident with no residual neurological deficits. Recent fasting blood glucose of $87 \mathrm{mg} /$ $\mathrm{dL}$ at a routine primary care office visit confirmed her nondiabetic status amid a body mass index (BMI) of $30.2 \mathrm{~kg} / \mathrm{m}^{2}$. The patient also had a negative family history for diabetes. The patient was given a trapezius trigger point injection of 1 $\mathrm{mL}$ of $4 \mathrm{mg} / \mathrm{mL}$ dexamethasone with $1 \mathrm{~mL}$ of $0.5 \%$ bupivacaine, and started on meloxicam, tramadol and methocarbamol. Three weeks later a repeat injection was administered. Plain X-ray film (Fig. 1) and MRI without contrast (Fig. 2) of the cervical spine were notable for moderate to severe degenerative disc disease at $\mathrm{C} 6-\mathrm{C} 7$ as well as severe narrowing of the left $\mathrm{C} 4-\mathrm{C} 5$ neuro-foramin. One week following the second trigger point injection, a pain management specialist administered $40 \mathrm{mg}$ of triamcinolone and $2 \mathrm{~mL}$ of $0.9 \%$ 


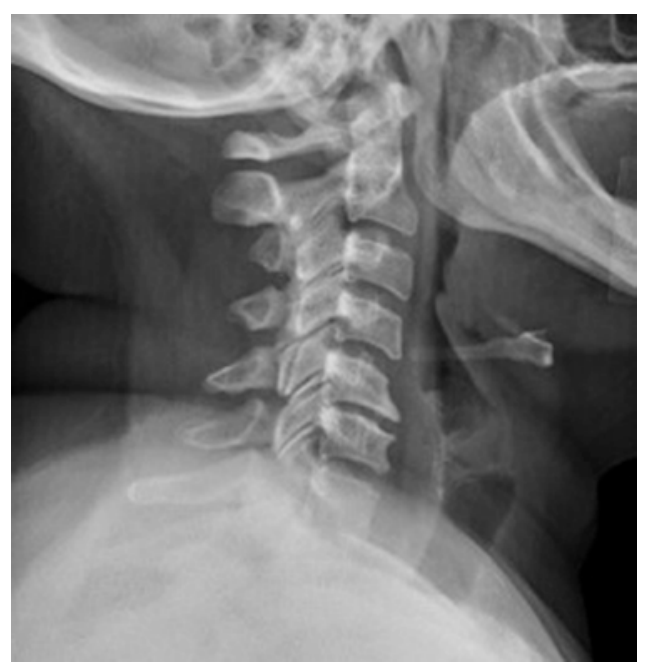

Figure 1. Cervical X-ray showing mild multilevel degenerative changes, especially at $\mathrm{C} 5-\mathrm{C} 6$, with no acute fracture or subluxation.

normal saline at the C6-C7 cervical inter-vertebral space under fluoroscopy. The patient presented to the emergency department 1 week after receiving the fluoroscopy-guided epidural injection, complaining of a 3-day history of xerostomia, polydipsia, polyuria, generalized weakness and diarrhea. Initial fingerstick glucose was $410 \mathrm{mg} / \mathrm{dL}$. Urinalysis showed glucose $>1,000 \mathrm{mg} / \mathrm{dL}$, ketones $15 \mathrm{mg} / \mathrm{dL}$ and positive nitrites. It was negative for leukocyte esterase and urine culture was notable for $>100,000 \mathrm{cfu} / \mathrm{mL}$ of E. coli. Chemistry studies showed a calculated anion gap of $15.6 \mathrm{mmol} / \mathrm{L}$, acetone of $1.2 \mathrm{mmol} / \mathrm{L}$ and potassium of 3.1 . The patient was started on an insulin drip with fluid resuscitation and admitted to the intensive care unit with a diagnosis of DKA.

\section{Discussion}

Our case describes a patient who was a documented non-diabetic who went into DKA following cervical epidural steroid injection. At the time of the epidural injection, the patient showed no signs of acute infection, concomitant drug use or any documented diabetes, all potential causes of DKA [6]. The patient had been seen regularly for chronic degenerative changes and had recent workup confirming her non-diabetic status. Three days after the fluoroscopy-guided epidural injection, the patient complained of increased urination, dry mouth, generalized weakness and diarrhea. Upon admission, the patient's physical exam and laboratory findings were diagnostic for DKA.

Studies have shown epidural steroid injections can transiently raise blood glucose levels in diagnosed diabetics [7]. Following epidural steroid injections, elevations in postprandial glucose levels have also been shown in non-diabetics, but the elevations lasted longer in diabetic than non-diabetic

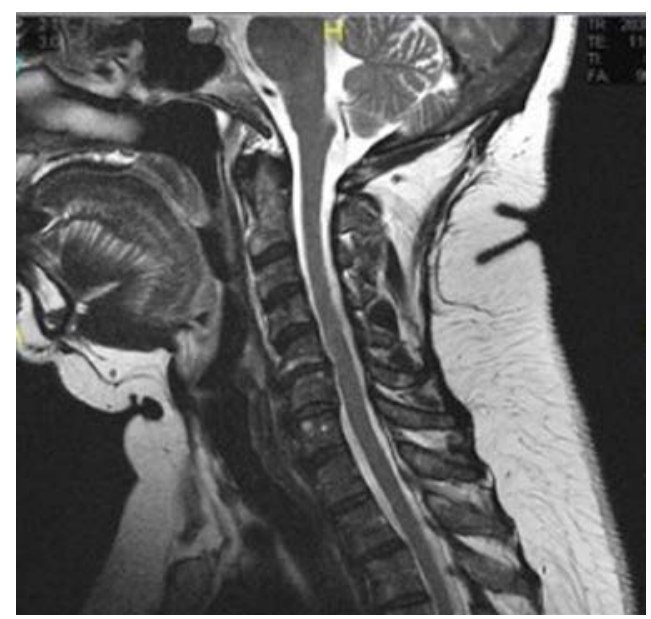

Figure 2. Sagittal T2 weighted MRI of the cervical spine without contrast showing severe degenerative disc disease at $\mathrm{C} 5-\mathrm{C} 6$ and $\mathrm{C} 6-\mathrm{C} 7$.

patients [8]. Although the mechanism of hyperglycemia following epidural steroid injection is clear, it remains difficult to distinguish how long these effects will last. Injection location (cervical, thoracic, lumbar), route of injection (epidural, intra-articular), dose and type of corticosteroid (triamcicolone, methylprednisolone) are all important prognostic variables. The duration of hyperglycemia and insulin resistance depends on the type of corticosteroid administered: triamcicolone (duration of action 7 - 84 days, half-life 5 - 8 days) versus methylprednisolone (duration of action 14 days, halflife 3.2 - 6.4 days) [9]. Our patient was administered a highdose $40 \mathrm{mg}$ triamcinolone injection and did not complain of any symptoms until 3 days after the injection. Because of the physiologic properties of this particular steroid involving the duration of action and half-life, it makes sense the patient did not have any symptoms until 3 days after the fluoroscopyguided injection. Both increased glucose levels and epidural steroid injections have each been shown to increase the risk of subsequent infection [7]. Even though any sort of infection, such as a urinary tract infection (UTI), increases the chances of a diabetic going into DKA, our postmenopausal patient with no history of recurrent UTI did not complain of classic UTI symptoms such as dysuria, urgency, suprapubic pain and/or hematuria, amid a positive urinary culture for nitrites and E. coli [10]. We suspect our patient's UTI was not the initial cause of her DKA, but likely colonization from asymptomatic bacteriuria.

Though uncommon, DKA can arise following either oral or epidural steroid treatments in diagnosed diabetics $[6,8]$. The literature does describe some cases of DKA arising in non-diabetics following steroid treatment, but many of these patients have a concurrent infection exacerbating the metabolic disorder by activating endogenous stress hormones [8, 11]. There are also cases of non-diabetic pregnant women administered glucocorticoids and subsequently presenting 
with DKA [12]. However, in this case, acute steroid-induced insulin resistance, superimposed on a pregnancy-related fasting insulinopenic state, may have served as the trigger [12]. Current literature lacks sufficient data on epidural steroid injections in non-diabetics and the occurrence of DKA. In addition, there is a dearth of case reports pertaining to this complication in this specific patient population. Our case is unique because our patient was a documented non-diabetic with no concomitant infection or any evidence of previous adverse effects to any prior epidural steroid injections.

The standard of care for managing DKA remains the same regardless of the cause: administration of insulin and correction of electrolyte abnormalities including hyperglycemia, hypovolemia, metabolic acidosis and potassium depletion. Though rare, DKA arising after an epidural steroid injection should warrant halting of any further epidural steroid injections until a fasting glucose assay 3 - 4 weeks following the initial injection confirms non-diabetic status [8]. If the patient turns out to be a previously undiagnosed diabetic, future epidural steroid injections should be met with caution as the possibility of a cumulative effect on insulin sensitivity arises because of frequent injections [13]. Patients with risk factors for diabetes must be screened according to guidelines prior to undertaking steroid injections. Additionally, they may need to be advised to seek earlier treatment for potential signs of infection as, just like those with systemic steroids, they are at higher risk for a non-specific time course following the injection. If no infection is found, the signs may be related to another complication, such as DKA, of the steroid injections. Clinicians should counsel diabetic patients about the importance of glucose control pre-injection and for at least the week following injection. Confirmed diabetics seeking epidural steroid injections should also be administered a smaller dose of corticosteroids as the efficacy remains the same despite the lower risk of elevated glucose levels following injection [14]. Even in confirmed non-diabetic patients, further epidural steroid injections should be met with caution as the possible effects of elevated glucose levels and insulin resistance remain [8]. Although epidural steroid injections remain a standard of care for degenerative disc disease in the aging population, clinicians must consider the myriad of complications that may arise from this treatment modality versus other more aggressive surgical interventions.

\section{References}

1. Ciol MA, Deyo RA, Howell E, Kreif S. An assessment of surgery for spinal stenosis: time trends, geographic variations, complications, and reoperations. J Am Geriatr Soc. 1996;44(3):285-290.
2. Beyaz SG, Eman A. Fluoroscopy guided cervical interlaminar steroid injections in patients with cervical pain syndromes: a retrospective study. J Back Musculoskelet Rehabil. 2013;26(1):85-91.

3. Abdi S, Datta S, Lucas LF. Role of epidural steroids in the management of chronic spinal pain: a systematic review of effectiveness and complications. Pain Physician. 2005;8(1):127-143.

4. Botwin KP, Castellanos R, Rao S, Hanna AF, Torres-Ramos FM, Gruber RD, Bouchlas CG, et al. Complications of fluoroscopically guided interlaminar cervical epidural injections. Arch Phys Med Rehabil. 2003;84(5):627-633.

5. Wang AA, Hutchinson DT. The effect of corticosteroid injection for trigger finger on blood glucose level in diabetic patients. J Hand Surg Am. 2006;31(6):979-981.

6. Kitabchi AE, Umpierrez GE, Murphy MB, Barrett EJ, Kreisberg RA, Malone JI, Wall BM. Management of hyperglycemic crises in patients with diabetes. Diabetes Care. 2001;24(1):131-153.

7. Even JL, Crosby CG, Song Y, McGirt MJ, Devin CJ. Effects of epidural steroid injections on blood glucose levels in patients with diabetes mellitus. Spine (Phila Pa 1976). 2012;37(1):E46-50.

8. Younes M, Neffati F, Touzi M, Hassen-Zrour S, Fendri Y, Bejia I, Ben Amor A, et al. Systemic effects of epidural and intra-articular glucocorticoid injections in diabetic and non-diabetic patients. Joint Bone Spine. 2007;74(5):472-476.

9. Catalano LW, 3rd, Glickel SZ, Barron OA, Harrison R, Marshall A, Purcelli-Lafer M. Effect of local corticosteroid injection of the hand and wrist on blood glucose in patients with diabetes mellitus. Orthopedics. 2012;35(12):e1754-1758.

10. Jackson SL, Boyko EJ, Scholes D, Abraham L, Gupta K, Fihn SD. Predictors of urinary tract infection after menopause: a prospective study. Am J Med. 2004;117(12):903-911.

11. Cagdas DN, Pac FA, Cakal E. Glucocorticoid-induced diabetic ketoacidosis in acute rheumatic fever. J Cardiovasc Pharmacol Ther. 2008;13(4):298-300.

12. Bedalov A, Balasubramanyam A. Glucocorticoid-induced ketoacidosis in gestational diabetes: sequela of the acute treatment of preterm labor. A case report. Diabetes Care. 1997;20(6):922-924.

13. Ward A, Watson J, Wood P, Dunne C, Kerr D. Glucocorticoid epidural for sciatica: metabolic and endocrine sequelae. Rheumatology (Oxford). 2002;41(1):68-71.

14. Kim WH, Sim WS, Shin BS, Lee CJ, Jin HS, Lee JY, Roe $\mathrm{HJ}$, et al. Effects of two different doses of epidural steroid on blood glucose levels and pain control in patients with diabetes mellitus. Pain Physician. 2013;16(6):557568 . 